\title{
Statistical Analysis of a Local Quadratic Criterion for Blind Speech Extraction
}

\author{
Benny Sällberg, Member, IEEE, Nedelko Grbić, Member, IEEE, and Ingvar Claesson, Member, IEEE
}

\begin{abstract}
This letter aims at complementing previous empirical work regarding a certain beamforming technique for blind speech extraction that uses a local quadratic approximation of a Kurtosis expression. It is shown here that the proposed method possesses a fixed-point property which means that it remains at an optimal solution once this solution has been reached. The proposed method's fixed-point property is valid for a range of source signals including Gaussian sources. This is an improvement over the FastICA method which diverges at the optimal points that correspond to a Gaussian source. In a real application, it cannot be assured that non-Gaussian mixtures are constantly observed; hence, the proposed method is a viable alternative in that case. The fixed-point property further implies that the approximative Kurtosis expression is identical to the true Kurtosis value at an optimal point which, in turn, means that the approximation error is zero. In addition, the convergence towards an optimal solution is always in the direction of a local minimum point even though the optimal solution that correspond to a super-Gaussian source is always a maximum solution which harmonizes with the concept of Kurtosis maximization.
\end{abstract}

Index Terms-Array signal processing, higher order statistics, speech enhancement.

\section{INTRODUCTION}

$\mathbf{S}$ PEECH extraction refers to the process of filtering a received mixture of acoustic signals of which at least one is a speech signal while the other signals are regarded as undesired noise, so that the speech must be extracted or, in fact, enhanced. Beamforming is a versatile speech extraction method as it can filter signals both in the temporal domain and in the spatial domain [1]. A blind adaptive beamformer is able to obtain the speech extraction effect gradually without any explicit references such as knowledge about the microphones' positions and the number and locations of acoustic sources [2]. Instead, some other assumption is made about the source signals [3], [4]. One such assumption is that source signals may carry different Kurtosis values, e.g., noise is often assumed to be Gaussian and having a zero Kurtosis value, while a speech signal generally carries a much higher Kurtosis value [5]. A blind beamformer can therefore be constructed by maximizing the Kurtosis value at the beamformer's output signal [6]. Approximative Newton methods, including a class of FastICA methods based on the Kurtosis contrast function (see, e.g., [7] and [8])

Manuscript received August 20, 2008; revised October 27, 2008. Current version published January 08,2009 . The associate editor coordinating the review of this manuscript and approving it for publication was Dr. Sergiy A. Vorobyov.

The authors are with the Department of Signal Processing, Blekinge Institute of Technology, SE-372 25 Ronneby, Sweden (e-mail: bsa@bth.se; ngr@bth.se; icl@bth.se).

Digital Object Identifier 10.1109/LSP.2008.2009839

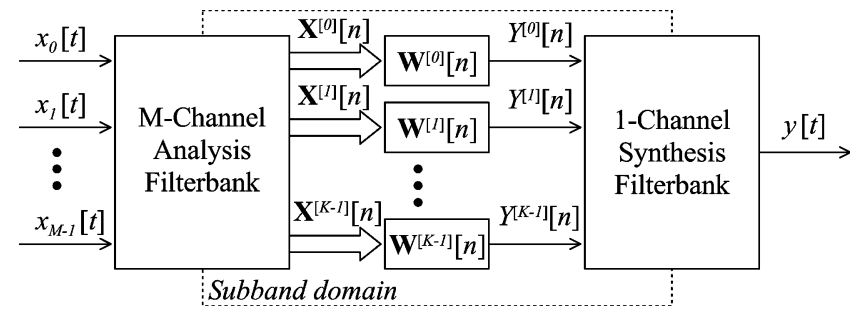

Fig. 1. Subband domain filter-and-sum beamformer for $M$ sensor signals and $K$ subbands.

and a closely related method [9], have been proposed in relation to this. However, the FastICA-like methods require a number of simplifications inside the optimization routine which make their approximations inexact at an optimum solution. A recently proposed technique approximates the Kurtosis value using a local quadratic function which is then solved at each iteration [10]-[12]. Robust methods for solving a quadratic problem exist in a rich variety today (see, e.g., [2]) which makes the proposed method practically tractable. Furthermore, the quadratic approximation of Kurtosis has been analyzed empirically in the past [10]-[12] and this method has shown itself capable of delivering a high level of speech extraction in various adverse environments. However, to date, no statistical analysis has been carried out for this particular approximation technique. The objective of this letter is to provide a statistical analysis of the quadratic Kurtosis criterion in order to explain some of its expected statistical behaviors and benefits.

The outline is as follows: The adopted system and the data model is described in Section II. The recently introduced approximation technique is provided in Section III and a blind beamformer based on this approximation technique is derived in Section IV. A statistical analysis is performed in Section V, and Section VI concludes this letter.

\section{SYSTEM AND DATA MODEL}

In this section, a convolution model is adopted where a set of source signals are emitted in a room and received by an array of microphones. The adopted filtering (beamformer) structure is commonly denoted as a filter-and-sum beamformer in the frequency domain [1], and the system model of the structure is illustrated in Fig. 1.

\section{A. System Model}

The system model assumes an array of $M$ microphones where each received real-valued time signal is denoted as $x_{m}[t]$ for $m=(0,1, \ldots, M-1)$, where $t$ represents continuous time. Each received time signal is sampled and decomposed into a 
time-frequency representation using a filter bank [13] with $K$ subbands, and where each microphone subband signal is denoted $X_{m}^{[k]}[n]$ with subband index $k=(0,1, \ldots, K-1)$ and where $n$ is a sample index in the subband domain. The reason for using a subband representation of the data is that a convolution model in the time domain corresponds to a multiplicative model in the frequency domain [14], [15]. The received signal vector is composed as $\mathbf{X}^{[k]}[n]=\mathbf{A}^{[k]} \mathbf{S}^{[k]}[n]$. The matrix $\mathbf{A}^{[k]}$ of size $M \times I$ is a source mixing matrix. The signal vector $\mathbf{S}^{[k]}[n]=\left(S_{0}^{[k]}[n], S_{1}^{[k]}[n], \ldots, S_{I-1}^{[k]}[n]\right)^{T}$ contains the $I$ original, independent source signals. The notation $(\cdot)^{T}$ denotes the vector transpose. The output signal $Y^{[k]}[n]$ of the beamformer is computed by filtering the received signal vector by the beamformer filter vector $\mathbf{W}^{[k]}[n]$, i.e., $Y^{[k]}[n]=\mathbf{W}^{[k]}[n]^{H} \mathbf{X}^{[k]}[n]$. The notation $(\cdot)^{H}$ denotes the complex conjugate vector transpose. This particular filtering uses one filter-tap per subband. While it is possible to extend the beamformer filter to include multiple filter-taps, it is not done here in order to simplify the statistical analysis. It is noted that, if $I>M$, an inverse to $\mathbf{A}^{[k]}$ does not exist in general and the best solution is found through a pseudo-inverse of $\mathbf{A}^{[k]}$. However, in order to conserve the readability in this letter, it is assumed that an inverse to $\mathbf{A}^{[k]}$ exists. The time-domain output signal $y[t]$ is then computed from the subband output signal $Y^{[k]}[n]$ by a synthesis filter bank matched to the analysis filter bank.

\section{B. Data Model}

The original sources are assumed to have unity power, i.e., $E\left\{\mathbf{S}^{[k]}[n] \mathbf{S}^{[k]}[n]^{H}\right\}=\mathbf{I}_{I}$, where $E\{\}$ represents the expectation operation, and $\mathbf{I}_{I}$ denotes an identity matrix of the size $I \times I$. Following [7] and [9], it is furthermore assumed that the sources have circular distributions, which implies that $E\left\{\mathbf{S}^{[k]}[n] \mathbf{S}^{[k]}[n]^{T}\right\}=\mathbf{0}_{I \times I}$. The matrix $\mathbf{0}_{I \times I}$ is a null-matrix of the size $I \times I$. Furthermore, the Kurtosis value of each source is $\kappa\left\{S_{i}^{[k]}[n]\right\}=E\left\{\left|S_{i}^{[k]}[n]\right|^{4}\right\}-2$.

\section{LOCAL QUADRATIC CRITERION}

In [10]-[12], a local quadratic criterion was derived for the purpose of Kurtosis maximization in a speech extraction application. The criterion was derived based on the inspiring paper by Yang regarding an iterative procedure for subspace tracking [16]. The key idea when constructing a quadratic approximation of a high order expression is to make use of the current filter weights $\mathbf{W}^{[k]}[n-1]$ to form an a priori output signal $\tilde{Y}^{[k]}[n]=\mathbf{W}^{[k]}[n-1]^{H} \mathbf{X}^{[k]}[n]$. Some output signal components $Y^{[k]}[n]$ are then replaced by this a priori output signal in the high order expression, e.g., the Kurtosis expression. In the end, this procedure renders an expression that is quadratic with regard to the new beamformer filter weights $\mathbf{W}^{[k]}[n]$.

To clarify further derivations, the Kurtosis value of the beamformer's output signal is notated as $\kappa\left\{\mathbf{W}^{[k]}[n]\right\} \equiv$ $\kappa\left\{Y^{[k]}[n]\right\}$, which is equal to

$$
\kappa\left\{\mathbf{W}^{[k]}[n]\right\}=E\left\{\left|Y^{[k]}[n]\right|^{4}\right\}-2 E\left\{\left|Y^{[k]}[n]\right|^{2}\right\}^{2} .
$$

This expression is obviously of order four with regard to $\mathbf{W}^{[k]}[n]$. Following [10]-[12], this Kurtosis expression can be approximated and rewritten into a quadratic form

$$
\begin{aligned}
& \hat{\kappa}\left\{\mathbf{W}^{[k]}[n]\right\}=E\left\{\left|\tilde{Y}^{[k]}[n]\right|^{2}\left|Y^{[k]}[n]\right|^{2}\right\} \\
& -2 E\left\{\left|\tilde{Y}^{[k]}[n]\right|^{2}\right\} \operatorname{Re}\left\{E\left\{\tilde{Y}^{[k]}[n]^{*} Y^{[k]}[n]\right\}\right\} .
\end{aligned}
$$

This expression is clearly quadratic with regard to $\mathbf{W}^{[k]}[n]$. In this expression, the notation ( $)^{*}$ refers to the complex conjugate, and $\operatorname{Re}\{\}$ takes the real part of its argument and it is employed in order to ensure that the approximative Kurtosis expression is real valued. The Brandwood gradient and Hessian [17] of (2) with regard to $\mathbf{W}^{[k]}[n]^{*}$ are

$$
\begin{array}{r}
\nabla \hat{\kappa}\left\{\mathbf{W}^{[k]}[n]\right\}=E\left\{\left|\tilde{Y}^{[k]}[n]\right|^{2} Y^{[k]}[n]^{*} \mathbf{X}^{[k]}[n]\right\} \\
-2 E\left\{\left|\tilde{Y}^{[k]}[n]\right|^{2}\right\} E\left\{\tilde{Y}^{[k]}[n]^{*} \mathbf{X}^{[k]}[n]\right\}
\end{array}
$$

and

$$
\nabla^{2} \hat{\kappa}\left\{\mathbf{W}^{[k]}[n]\right\}=E\left\{\left|\tilde{Y}^{[k]}[n]\right|^{2} \mathbf{X}^{[k]}[n] \mathbf{X}^{[k]}[n]^{H}\right\} .
$$

There may be other ways to approximate the fourth-order Kurtosis expression that are not outlined here regarding which terms $Y^{[k]}[n]$ are exchanged with $\tilde{Y}^{[k]}[n]$ in the approximation. Such reformulations can be the focus of future research.

\section{BLIND ADAPTIVE BEAMFORMING}

The proposed blind adaptive beamformer continuously updates its beamformer weights $\mathbf{W}^{[k]}[n]$ in order to obtain a maximization of the output signal's Kurtosis value. While the approximative Kurtosis expression (2) is quadratic with regard to $\mathbf{W}^{[k]}[n]$, its optimal solution is found at a point where the gradient with regard to $\mathbf{W}^{[k]}[n]$ evaluates to zero, i.e., $\nabla \hat{\kappa}\left\{\mathbf{W}^{[k]}[n]\right\}=\mathbf{0}_{M \times 1}$. However, experiments on real data have shown that this direct approach leads to a highly fluctuating solution with a degraded speech quality as a result. A viable approach to reduce the fluctuations is by using a smoothed filter update equation

$$
\begin{aligned}
\mathbf{W}_{+}= & \mathbf{W}^{[k]}[n-1]-\gamma^{[k]} E\left\{\left|\tilde{Y}^{[k]}[n]\right|^{2}\right\} \\
& \cdot E\left\{\left|\tilde{Y}^{[k]}[n]\right|^{2} \mathbf{X}^{[k]}[n] \mathbf{X}^{[k]}[n]^{H}\right\}^{-1} \\
& \cdot E\left\{\tilde{Y}^{[k]}[n]^{*} \mathbf{X}^{[k]}[n]\right\}
\end{aligned}
$$

where $0<\gamma^{[k]} \leq 1$ is a smoothing parameter. A temporary vector $\mathbf{W}_{+}$is used in order to compute a unity-norm constraint for the updated filter coefficients as $\mathbf{W}^{[k]}[n]=\mathbf{W}_{+} /\left\|\mathbf{W}_{+}\right\|_{2}$.

When the algorithm has converged to an optimal solution, it is expected that $\mathbf{W}^{[k]}[n]=\mathbf{W}^{[k]}[n-1]$. If this is the case, $Y^{[k]}[n]=\widetilde{Y}^{[k]}[n]$, which in turn yields that $\hat{\kappa}\left\{\mathbf{W}^{[k]}[n]\right\}=\kappa\left\{\mathbf{W}^{[k]}[n]\right\}$, i.e., the introduced approximative Kurtosis value is equal to the true Kurtosis value. The next section will show that the quadratic method has a fixed-point property which means that the method stays at an 
optimal solution when it finds one, which, in turn, yields that the approximation error is zero. In addition, an analysis of the local consistency will show that the Hessian matrix is negative definite around an optimal solution. The update (5) has to compensate for the negative definiteness by a negative update direction.

\section{Statistical Analysis}

In [7] and [9], a statistical analysis was used for a FastICA method and a closely related method. A similar analysis is used here for the local quadratic Kurtosis criterion.

First, a notation strategy is adopted in order to clarify the presentation. A variable that represents a priori information is designated by the " $\sim$ "-sign. In addition, while the statistical analysis is performed for one subband $k$ and at a point in time $n$, these indices are omitted in the sequel.

It is assumed that the received signal vector is made uncorrelated prior to the analysis using a principal component analysis (PCA) preprocessing stage. The PCA decorrelation has the same impact as if the mixing matrix $\mathbf{A}$ would possess a Unitary property, i.e., $E\left\{\mathbf{X X}^{H}\right\}=\mathbf{A} E\left\{\mathbf{S S}^{H}\right\} \mathbf{A}^{H}=\mathbf{A} \mathbf{A}^{H}=\mathbf{I}_{M}$.

Two properties of the blind beamformer are analyzed: the fixed-point property and the local consistency. If an algorithm possesses a fixed-point property, it means that the algorithm stays at an optimal solution when it finds one. The local consistency is used to analyze the algorithm behavior around an optimal solution. It is thus possible to, for instance, determine what kind of local optimal solution it is (a maximal solution, a minimal solution, or a saddle point).

\section{A. Fixed-Point Behavior}

In order to analyze the fixed-point behavior, it is assumed that the algorithm has found an optimal solution that extracts the $i$ th source, i.e., $\widetilde{\mathbf{W}}=\mathbf{W}_{\mathrm{opt}}$ so that $\widetilde{Y}=\mathbf{W}_{\mathrm{opt}}^{H} \mathbf{X}=S_{i}$. The filter vector update equation is therefore equal to

$$
\mathbf{W}_{+}=\widetilde{\mathbf{W}}-\gamma E\left\{\left|S_{i}\right|^{2}\right\} E\left\{\left|S_{i}\right|^{2} \mathbf{X} \mathbf{X}^{H}\right\}^{-1} E\left\{S_{i}^{*} \mathbf{X}\right\}
$$

The various statistical expectations above are evaluated as

$$
\begin{aligned}
E\left\{\left|S_{i}\right|^{2}\right\} & =1 \\
E\left\{\left|S_{i}\right|^{2} \mathbf{X X}^{H}\right\}^{-1} & =\mathbf{A} \Lambda_{i}^{-1} \mathbf{A}^{H} \\
E\left\{S_{i}^{*} \mathbf{X}\right\} & =\mathbf{A} \mathbf{u}_{i} .
\end{aligned}
$$

The matrix $\Lambda_{i}$ (size $\left.I \times I\right)$ is a diagonal matrix, whose diagonal entries have the value one, except for the $i$ th diagonal element that has the value $\kappa\left\{S_{i}\right\}+2$. The vector $\mathbf{u}_{i}$ is a vector with zerovalued elements except for at the position $i$ where the element has the value one. The filter vector update equation is

$$
\mathbf{W}_{+}=\widetilde{\mathbf{W}}-\gamma \mathbf{A} \Lambda_{i}^{-1} \mathbf{u}_{i}=\widetilde{\mathbf{W}}-\frac{\gamma}{\kappa\left\{S_{i}\right\}+2} \mathbf{A} \mathbf{u}_{i} .
$$

The optimal solution is such that $\mathbf{W}_{\mathrm{opt}}^{H} \mathbf{A S}=S_{i}$; hence, $\mathbf{u}_{i}=$ $\mathbf{A}^{H} \mathbf{W}_{\text {opt }}$, and therefore, $\mathbf{W}_{\text {opt }}=\mathbf{A} \mathbf{u}_{i}$, which yields that

$$
\mathbf{W}_{+}=\left(1-\frac{\gamma}{\kappa\left\{S_{i}\right\}+2}\right) \mathbf{W}_{\mathrm{opt}} .
$$

Hence, provided that $1-\gamma /\left(\kappa\left\{S_{i}\right\}+2\right) \neq 0$, i.e., $\gamma \neq \kappa\left\{S_{i}\right\}+$ 2 , it is clear that the blind beamformer (5) possesses the fixedpoint property

$$
\mathbf{W}=\frac{\mathbf{W}_{+}}{\left\|\mathbf{W}_{+}\right\|_{2}}= \pm \mathbf{W}_{\mathrm{opt}} .
$$

While $0<\gamma \leq 1$, there is only one extreme case where the algorithm does not possess the fixed-point property, i.e., $\gamma=$ $\kappa\left\{S_{i}\right\}+2$, namely, if $-2<\kappa\left\{S_{i}\right\} \leq-1$, which implies that the source $S_{i}$ is sub-Gaussian. In all other cases, the algorithm possesses a fixed-point property that is also valid for a Gaussian source $\left(\kappa\left\{S_{i}\right\}=0\right)$.

\section{B. Local Consistency}

A second-order Taylor series expansion is computed at a point $\mathbf{W}_{\text {opt }}+\mathbf{P}$ around the optimal solution $\mathbf{W}_{\text {opt }}$, where $\mathbf{P}$ is a small perturbation vector (the term "small" implies that $\|\mathbf{P}\|_{2} \ll 1$ ), according to

$$
\begin{aligned}
\hat{\kappa}\left\{\mathbf{W}_{\text {opt }}+\mathbf{P}\right\}= & \hat{\kappa}\left\{\mathbf{W}_{\text {opt }}\right\}+2 \operatorname{Re}\left\{\mathbf{P}^{H} \nabla \hat{\kappa}\left\{\mathbf{W}_{\text {opt }}\right\}\right\} \\
& +\mathbf{P}^{H} \nabla^{2} \hat{\kappa}\left\{\mathbf{W}_{\text {opt }}\right\} \mathbf{P} .
\end{aligned}
$$

The gradient vector $\nabla \hat{\kappa}\left\{\mathbf{W}_{\text {opt }}\right\}$ is

$$
\begin{aligned}
\nabla \hat{\kappa}\left\{\mathbf{W}_{\mathrm{opt}}\right\}= & E\left\{\left|S_{i}\right|^{2} \mathbf{X} \mathbf{X}^{H}\right\} \mathbf{W}_{\mathrm{opt}} \\
& -2 E\left\{\left|S_{i}\right|^{2}\right\} E\left\{S_{i}^{*} \mathbf{X}\right\}= \\
= & \mathbf{A} \mathbf{\Lambda}_{i} \mathbf{u}_{i}-2 \mathbf{A} \mathbf{u}_{i}= \\
= & \kappa\left\{S_{i}\right\} \mathbf{W}_{\mathrm{opt}} .
\end{aligned}
$$

The Hessian matrix $\nabla^{2} \hat{\kappa}\left\{\mathbf{W}_{\text {opt }}\right\}$ is evaluated as

$$
\nabla^{2} \hat{\kappa}\left\{\mathbf{W}_{\text {opt }}\right\}=E\left\{\left|S_{i}\right|^{2} \mathbf{X X} \mathbf{X}^{H}\right\}=\mathbf{A} \mathbf{\Lambda}_{i} \mathbf{A}^{H} .
$$

Inserting (14) and (15) into (13) yields

$$
\begin{aligned}
\hat{\kappa}\left\{\mathbf{W}_{\text {opt }}+\mathbf{P}\right\}=\hat{\kappa}\left\{\mathbf{W}_{\text {opt }}\right\}+2 \kappa\left\{S_{i}\right\} R e\left\{\mathbf{P}^{H} \mathbf{W}_{\text {opt }}\right\} \\
\\
+\mathbf{P}^{H} \mathbf{A} \mathbf{\Lambda}_{i} \mathbf{A}^{H} \mathbf{P} .
\end{aligned}
$$

The unity norm constraint renders the relationship $2 R e\left\{\mathbf{P}^{H} \mathbf{W}_{\text {opt }}\right\}=-\mathbf{P}^{H} \mathbf{P}$ that yields

$$
\begin{gathered}
\hat{\kappa}\left\{\mathbf{W}_{\mathrm{opt}}+\mathbf{P}\right\}=\hat{\kappa}\left\{\mathbf{W}_{\mathrm{opt}}\right\}-\left(\kappa\left\{S_{i}\right\}-1\right) \mathbf{P}^{H} \mathbf{P} \\
+\left(\kappa\left\{S_{i}\right\}+1\right) \mathbf{P}^{H} \mathbf{A} \boldsymbol{\Delta}_{i} \mathbf{A}^{H} \mathbf{P} .
\end{gathered}
$$

The matrix $\boldsymbol{\Delta}_{i}$ (size $\left.I \times I\right)$ has elements that are zero-valued except for a single element at position $(i, i)$ which has the value one. Obviously, $0 \leq \mathbf{P}^{H} \mathbf{A} \boldsymbol{\Delta}_{i} \mathbf{A}^{H} \mathbf{P} \leq \mathbf{P}^{H} \mathbf{P}$, which equals $\mathbf{P}^{H} \mathbf{A} \boldsymbol{\Delta}_{i} \mathbf{A}^{H} \mathbf{P}=\alpha \mathbf{P}^{H} \mathbf{P}$ for a factor $\alpha \in[0,1]$

$$
\begin{aligned}
\hat{\kappa}\left\{\mathbf{W}_{\mathrm{opt}}+\mathbf{P}\right\}=\hat{\kappa}\left\{\mathbf{W}_{\mathrm{opt}}\right\} \\
-\left((1-\alpha)\left(\kappa\left\{S_{i}\right\}+2\right)-3+\alpha\right) \mathbf{P}^{H} \mathbf{P} .
\end{aligned}
$$

While $\mathbf{P}^{H} \mathbf{P} \geq 0$, the type of optimal solution $\mathbf{W}_{\text {opt }}$ is dependent on the relationship between $\alpha$ and the Kurtosis value $\kappa\left\{S_{i}\right\}$ through the sign of $(1-\alpha)\left(\kappa\left\{S_{i}\right\}+2\right)-3+\alpha$. This relationship is shown in Fig. 2. An optimal solution that corresponds to a source with the Kurtosis value $-2<\kappa\left\{S_{i}\right\}<1$ (this includes a Gaussian source with $\kappa\left\{S_{i}\right\}=0$ ) is always, independent of 


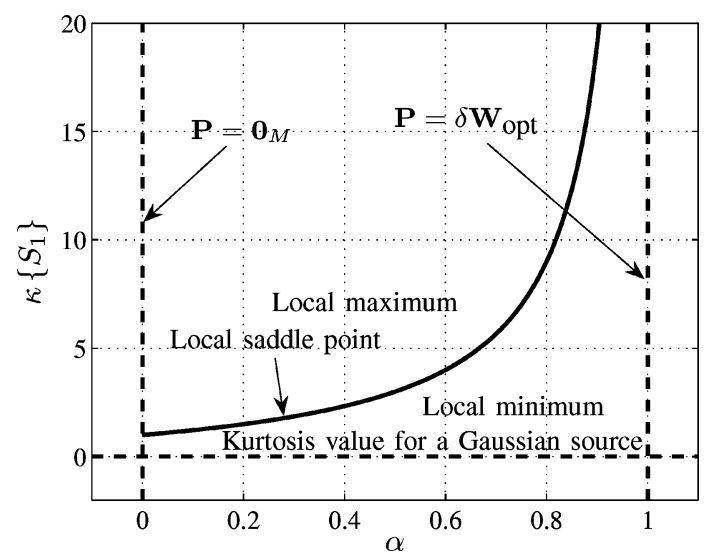

Fig. 2. Solid curve shows the region where the solution is a saddle point, a maximum solution (points above the curve), and a minimum solution (points below the curve).

the value of $\alpha$, a local minimum solution. An optimal solution corresponding to a super-Gaussian source, with $\kappa\left\{S_{i}\right\}>1$, can be any kind of local optimum (maximum, minimum, or saddle point) depending on the value of $\alpha$. However, when the algorithm starts to converge towards an optimal solution, it implies that $\alpha \approx 1$, independent of what Kurtosis value the source carries. This becomes clear if it is assumed that $\mathbf{P}=\delta \mathbf{W}_{\text {opt }}$, for a value $\delta>0$, while $\alpha=1$. Hence, the Hessian matrix corresponding to (2) is negative definite, and the algorithm always converges towards a local minimum solution. In order to compensate for the negative definiteness of the Hessian matrix, it is necessary for the algorithm to update in a direction opposite to a point where the gradient evaluates to zero in (5). However, provided that $\gamma \neq \kappa\left\{S_{i}\right\}+2$, the fixed-point property renders $\mathbf{P}=\mathbf{0}_{M}$ (i.e., $\alpha=0$ ) at an optimal solution. An optimal solution that corresponds to a super-Gaussian source $\left(\kappa\left\{S_{i}\right\}>1\right)$ is always a maximum solution which harmonizes with the concept of Kurtosis maximization.

\section{CONCLUSIONS}

A recent method for adaptive beamforming applied to blind speech extraction was proposed in [10]-[12]. The method uses an approximation of the fourth-order Kurtosis value in order to construct a local quadratic expression. The proposed method is practically viable while the quadratic approximation can be solved using standard methods, e.g., the recursive least squares (RLS) [2], [16]. Several empirical analyses was conducted in [10]-[12], and they showed the method's ability to extract speech in adverse environments. A statistical analysis of this method is provided in this letter with respect to the fixed-point property and the local consistency. It is shown that the proposed method possesses a fixed-point property if the optimal solution does not correspond to a sub-Gaussian source, i.e., with a Kurtosis value in the range $-2<\kappa\left\{S_{i}\right\} \leq-1$. This behavior is important in a real application where it cannot always be guaranteed that non-Gaussian sources are active, and it may be contrasted with the popular FastICA method (see, e.g., [7] and [9]) that has been shown to diverge for a Gaussian source, i.e., $\kappa\left\{S_{i}\right\}=0$. The fixed-point property also tells us that once the algorithm has converged to an optimal solution, it stays at this optimal solution. This renders that the introduced quadratic approximation of Kurtosis is equal to the true Kurtosis value at an optimal solution, i.e., the approximation error is equal to zero at an optimal solution. In addition, it is shown that the introduced quadratic approximation renders a local minimum in the direction towards an optimal solution. This means that the Hessian matrix of (2) is negative definite and the optimization approach results in a minimization strategy instead of a maximization strategy, even though the optimal solution that corresponds to a super-Gaussian source $\left(\kappa\left\{S_{i}\right\}>1\right)$ is always a maximum solution which harmonizes with the concept of Kurtosis maximization.

\section{REFERENCES}

[1] D. Johnson and D. Dudgeon, Array Signal Processing-Concepts and Techniques. Englewood Cliffs, NJ: Prentice-Hall, 1993.

[2] S. Haykin, Adaptive Filter Theory. New York: Wiley, 2002.

[3] A. Hyvärinen, J. Karhunen, and E. Oja, Independent Component Analysis. New York: Wiley, 2001.

[4] A. Cichocki and S. Amari, Adaptive Blind Signal and Image Processing-Learning Algorithms and Applications. New York: Wiley, 2003.

[5] W. Zhang and S. Gazor, "Statistical modelling of speech signals," in Proc. IEEE Int. Conf. Signal Processing, 2002, vol. 1, pp. 480-483.

[6] Z. Ding, "A new algorithm for automatic beamforming," in Proc. IEEE Asilomar Conf. Signals, Systems and Computers, 1991, vol. 2, pp. 689-693.

[7] E. Bingham and A. Hyvärinen, "A fast fixed-point algorithm for independent component analysis of complex valued signals," Int. J. Neural Syst., vol. 10, no. 1, pp. 1-8, 2000.

[8] S. C. Douglas, "Fixed-point FastICA algorithms for the blind separation of complex-valued signal mixtures," in Proc. IEEE Asilomar Conf. Signals, Systems and Computers, 2005, pp. 1320-1325.

[9] B. Sällberg, N. Grbić, and I. Claesson, "Complex-valued independent component analysis for online blind speech extraction," IEEE Trans. Audio, Speech, Lang. Process., vol. 16, no. 8, pp. 1624-1632, Nov. 2008.

[10] B. Sällberg, N. Grbić, and I. Claesson, "Online maximization of subband Kurtosis for blind adaptive beamforming in realtime speech extraction," in Proc. IEEE 15th Int. Conf. Digital Signal Processing, Jul. 2007, pp. 603-606.

[11] B. Sällberg, N. Grbić, and I. Claesson, "Online blind speech extraction based on a locally quadratic Kurtosis criteria and a preprocessing automatic gain controller," in Proc. IEEE 49th Int. Symp. ELMAR, Sep. 2007, pp. 139-142.

[12] B. Sällberg, N. Grbić, and I. Claesson, "An adaptive blind beamformer with an integrated single-channel noise reduction method for robust realtime blind speech extraction," in Proc. IEEE Int. Conf. Acoustic, Speech and Signal Processing, Mar. 2008, pp. 309-312.

[13] P. P. Vaidyanathan, Multirate Systems and Filter Banks. Englewood Cliffs, NJ: Prentice-Hall, 1993.

[14] P. Smaragdis, "Blind separation of convolved mixtures in the frequency domain," Elsevier Neurocomput., vol. 22, no. 1-3, pp. 21-34, 1998.

[15] N. Grbić, X.-J. Tao, S. E. Nordholm, and I. Claesson, "Blind signal separation using overcomplete subband representation," IEEE Trans. Speech Audio Process., vol. 9, no. 5, pp. 524-533, Jul. 2001.

[16] B. Yang, "Projection approximation subspace tracking," IEEE Trans. Signal Process., vol. 43, no. 1, pp. 95-107, Jan. 1995.

[17] D. H. Brandwood, "A complex gradient operator and its application in adaptive array theory," Proc. Inst. Elect. Eng., vol. 130, no. 1, pp. 11-16, Feb. 1983. 